\title{
Prevalence of Preoperative Anemia in Patients Admitted At Khartoum Teaching Dental Hospital
}

\author{
Amel Salah Eltayeb ${ }^{1}$, Manar E. Abdel-Rahman ${ }^{2}$, Ahmed M. Suleiman ${ }^{3}$ \\ ${ }^{I}$ (Department Of Oral And Maxillofacial Surgery, Khartoum Teaching Dental Hospital, Sudan) \\ ${ }_{2}^{2}$ (Department Of Statistics, Faculty Of Mathematical Sciences, University Of Khartoum, Sudan) \\ ${ }_{3}^{3}$ (Department Of Oral And Maxillofacial Surgery, Faculty Of Dentistry, University Of Khartoum, Sudan)
}

\begin{abstract}
:
Objective: The aim of this study was to evaluate preoperative anemia in patients undergoing oral and maxillofacial surgery under general anesthesia.

Material and methods: A prospective cross sectional hospital-based study was carried at Khartoum Teaching Dental Hospital. Five hundred and twenty three patients were included in the study, 324were males and 199 were females. Patients' age was ranging from 6 months to 84 years. Children formed $34.6 \%$ of the patients. Cleft lip and palate constituted the largest number of the patients (173). There were 70 oral cancer patients, 126 trauma patients and 154 patients with benign lesions. Anemia was defined according to the WHO criteria as hemoglobin concentration $(\mathrm{Hb})$ less than $13 \mathrm{~g} / \mathrm{dl}$ for males, less than $12 \mathrm{~g} / d l$ for females while Children were diagnosed based on their age. Variables related to hemoglobin level, age, gender and related diseases were analyzed. Test of significance $(P<0.05)$ was applied using SPSS version 20.

Results: Preoperative investigations revealed a high prevalence of anemia in patients constituting $48.2 \%$ for men, $45 \%$ for females and $47 \%$ for children. Severe anemia was found in $4.5 \%$ of children and $1.4 \%$ of males. The mean hemoglobin level for males was $12.8 \pm 2.1 \mathrm{~g} / \mathrm{dl}$, for females was $12.1 \pm 1.4$ and for children was 11.4 \pm 1.6 . One way analysis of variance revealed a significant difference in the mean of hemoglobin between patients with different diseases $(P$-value $=0.001)$.

Conclusion: The findings of this study indicated that anemia is a common finding among patients undergoing oral and maxillofacial surgery and most of the time it was discovered during the preoperative investigation for surgery preparation.
\end{abstract}

Keywords: Anemia, general anesthesia, oral cancer, cleft lip and palate, oral surgery.

\section{Introduction}

Anemia is common in patients undergoing major surgery(1). Although diagnosis and treatment of anemia preoperatively is essential to optimize the patient's condition(2), preoperative anemia treatment is not a priority for most surgeons(3). One third of all cases of anemia in older subjects result from nutritional inadequacy, one third results from chronic disease including chronic kidney disease, and the remaining cases of anemia are of undetermined etiology(4).

In 1995, nutritional anemia was among the ten major causes for hospital admission in Sudan (5). In kassala, east sudan; Abdalla et al, 2011 reported that the prevalence of anemia among adult is $36.2 \%$, regardless of their age, sex and educational level(6). A recent study in 2015 by Mohamed et al, conducted in Rural Schoolaged Sudanese Children found that prevalence of anemia was 29.7\%(7). Spahn et a, in a systematic review of some cohorts studies revealed that preoperative anemia is present among $50 \%$ of patients undergoing surgical hip fracture repair and is increased to $87 \%$ postoperatively(8). Another hospital based study by Vasudevaru et al, in India revealed $63 \%$ of oral cancer patients were anemic before treatment (9).

The aim of this study was to evaluate preoperative anemia in patients undergoing oral and maxillofacial surgery under general anesthesia.

\section{Material And Methods}

This is an observational cross sectional hospital based study. The study was conducted at Khartoum Teaching Dental Hospital (KTDH) between December 2013 and December 2014. All patients scheduled for operation under general anesthesia who met the inclusion criteria were included in the study. The exclusion criteria were: 1 . Patients who had received blood transfusion within the last three months or had any treatment for anemia. 2. Patients with history of surgery, chemotherapy or radiotherapy. 3. Patients with recurrent malignancy. 4. Patients with disease other than the cause (disease) of admission. All admitted patients were screened for eligibility criteria. Later investigations and demographic data were collected. Recruitment was done serially in one year duration until the sample size was attained. 
Anemia defined according to the WHO criteria (10), for males as haemoglobin concentration $(\mathrm{Hb})$ less than $13 \mathrm{~g} / \mathrm{dl}$, while for females, it was defined as less than $12 \mathrm{~g} / \mathrm{dl}$. Children were diagnosed based on their age. All eligible patients' data were extracted from the patients file then transferred to specific designed case record form. The assessment and data collection was carried by the principal investigator. Data were entered using Microsoft excel 2007, cleaned and analyzed using Statistical Package for Social Sciences (IBM SPSS Inc., Chicago, version (no.20) software. Data were summarized in frequency tables and pie charts. Categorical variables were reported as proportion and were compared using chi squared test. Continuous data were described as means (standard deviation). Approval obtained from the ethics committee ministry of health and information was treated with confidentiality

\section{Results}

Patients included in this study were 523 patients, 324(62\%) were males and $199(38 \%)$ were females. Patients' age was ranging from 6 months to 84 years and the mean age was 25.3 years. There were $181(34.6 \%)$ children in this study and $44.8 \%$ of those children were below five years. Oral cancer patients were $70(13.4 \%)$ of all the selected sample of patients followed by $126(24.1 \%)$ and $154(29.4 \%)$ for trauma patients and patients diagnosed with benign lesions (others), respectively. Cleft lip and palate formed the highest percentage of the patients $173(33.1 \%)$.

The mean of hemoglobin level was different between age groups. It was also different between males and females within the same age group as shown in table1.

Table 1: The relation between mean of hemoglobin, age and gender

\begin{tabular}{|l|c|c|c|c|l|l|l|l|}
\hline \multirow{2}{*}{} & \multicolumn{2}{|l}{ Oral cancer } & \multicolumn{2}{l|}{$\begin{array}{l}\text { Cleft lip And } \\
\text { palate }\end{array}$} & \multicolumn{2}{l|}{ trauma } & \multicolumn{2}{l|}{ Benign lesion } \\
\cline { 2 - 9 } & male & female & male & female & male & female & male & female \\
\hline $0-14$ yrs & - & - & 11.3 & 11.1 & 11.9 & 12.9 & 11.8 & 11.6 \\
\hline $15-24 y r s$ & - & 12.3 & 12.7 & 13.3 & 12.7 & 11.9 & 12.0 & 13.6 \\
\hline $25-34 y r s$ & 11.7 & - & 13.7 & 12.6 & 12.5 & 11.9 & 12.5 & 12.9 \\
\hline $35-44 y r s$ & 12.1 & 13.8 & 14.0 & 12.1 & 12.5 & 13.1 & 12.8 & 12.7 \\
\hline 45-54yrs & 11.8 & 12.6 & - & - & 14.3 & 10.9 & 09.6 & 13.7 \\
\hline $55+y r s$ & 12.2 & 12.4 & - & - & 11.3 & 13.3 & 12.2 & 12.0 \\
\hline
\end{tabular}

The overall mean of hemoglobin among each disease was different as shown in figure1. There was significant difference between groups ( $p$-value $=0.001$ ) when one way analysis of variance was done.

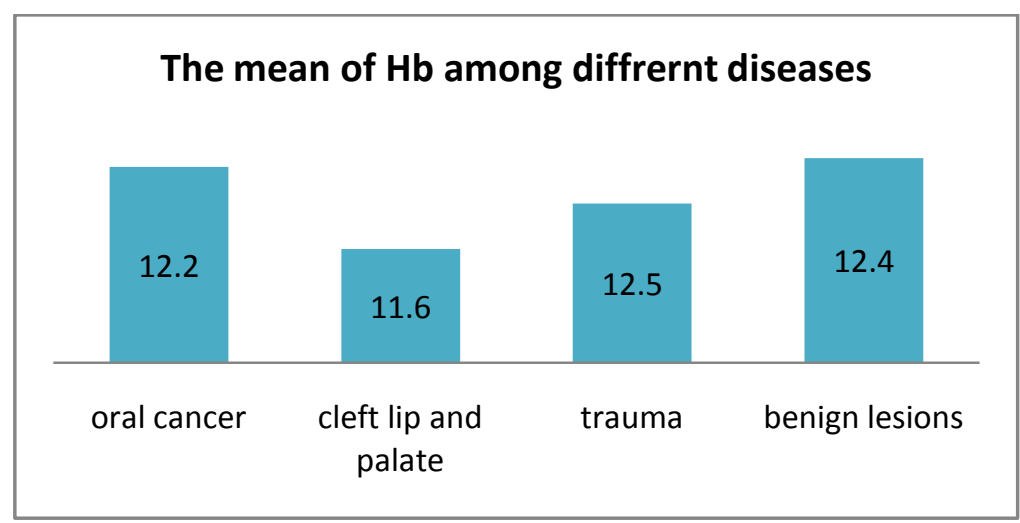

Figure 1: the mean of hemoglobin among different diseases

A comparison of hemoglobin level by diagnosis (disease) was done (bonferroni test) and the result showed a statistically significant difference in hemoglobin level between cleft patients and trauma patients $(\mathrm{p}-$ value<0.001).

A difference in hemoglobin level between cleft patients and patients with benign lesions (others) is found to be statistically significant ( $\mathrm{p}$-value $=0.001$ ) while it's not statistically significant in oral cancer patients with either cleft patients or patients with benign lesions (others). Table2. 
Table 2: comparison of hemoglobin level by diagnosis (diseases)

\begin{tabular}{|l|c|l|l|}
\hline Col mean & Oral cancer & Cleft patients & trauma \\
\hline Cleft patients & -0.595 & & \\
& 0.151 & 0.898 & \\
Trauma & 0.303 & $\underline{0.000}$ & \\
& 1.000 & 0.803 & -0.094 \\
Others & 0.209 & $\underline{0.001}$ & 1.000 \\
& 1.000 & & \\
\hline
\end{tabular}

In this study, there were 216 males; the mean age was 33 years. The chi square test was done to correlate age with diseases and results are statistically significant (p-value=0.000). In males, the mean of hemoglobin was $12.8 \pm 2.1$ and anemia was found in $48.2 \%$ of males. The chi square test was done to correlate anemia with diseases and results were statistically insignificant $(\mathrm{p}$-value $=0.309)$. Table 3

Table 3: Correlation between anemia and diseases among males

\begin{tabular}{|l|l|l|l|l|c|}
\hline hemoglobin & Oral cancer\% & Cleft lip/palate\% & Trauma\% & Others\% & Total sample \\
\hline Non-anemic & 17.1 & 12.6 & 39.6 & 30.6 & $111(100 \%)$ \\
\hline Mild anemia & 22.2 & 9.5 & 47.6 & 20.6 & $63(100 \%)$ \\
\hline Moderate anemia & 15.4 & 10.3 & 59 & 15.4 & $39(100 \%)$ \\
\hline Severe anemia & 0 & 0 & 100 & 0 & $3(100 \%)$ \\
\hline Total & 18.1 & 11.1 & 46.3 & 24.5 & $216(100 \%)$ \\
\hline
\end{tabular}

Pearson $\operatorname{chi}^{2}(9) 10.5301 \mathrm{Pr}=0.309$

There were 129 females in this study. The mean age was 38.4 years and the mean hemoglobin level was 12.1 \pm 1.4 . Chi square test was done to correlate age with disease and result was found to be statistically significant ( $\mathrm{p}$ value $=0.000$ ). In females, the mean of hemoglobin was $12.1 \pm 1.4$. Anemia was found in $45 \%$ of females. The chi square test was done to correlate anemia with diseases and results were statistically insignificant ( $\mathrm{p}$-value $=0.309)$. Table 4

Table 4: Correlation between anemia and diseases among males

\begin{tabular}{|l|l|l|l|l|c|}
\hline hemoglobin & $\begin{array}{l}\text { Oral } \\
\text { cancer\% }\end{array}$ & $\begin{array}{l}\text { Cleft } \\
\text { lip/palate\% }\end{array}$ & Trauma\% & Others\% & Total sample \\
\hline Non-anemic & 19.7 & 18.3 & 16.9 & 45.1 & $71(100 \%)$ \\
\hline Mild anemia & 25.7 & 11.4 & 8.6 & 54.3 & $35(100 \%)$ \\
\hline Moderate anemia & 34.8 & 4.3 & 17.4 & 43.5 & $23(100 \%)$ \\
\hline Severe anemia & 0 & 0 & 0 & 0 & $0(0 \%)$ \\
\hline Total & 24 & 14 & 14.7 & 47.3 & $129(100 \%)$ \\
\hline
\end{tabular}

Pearson chi2(6)6.090 $\mathrm{Pr}=0.413$

Children constituted $34.6 \%$ (181) of all patients and $61.3 \%$ of them were males. The most common disease in children was cleft lip and palate forming $74 \%$ of children. Figure 2.

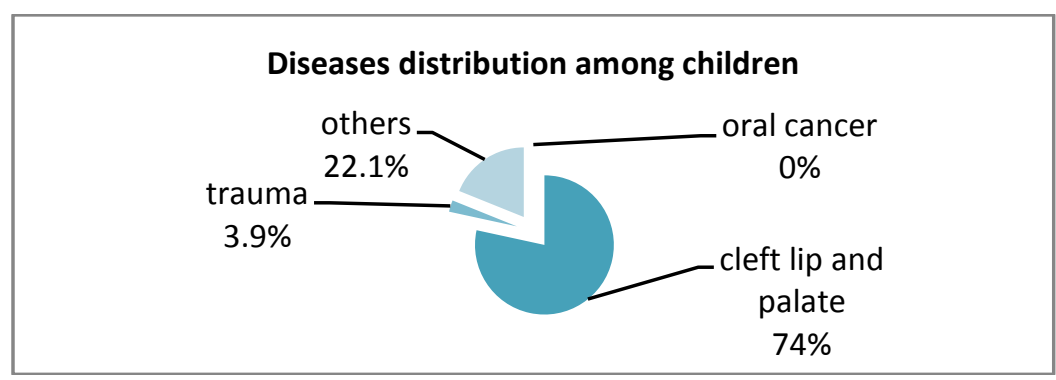

The correlation between age and disease was found to be statistically significant when chi square test was done (p-value $=0.000$ ) Table 5. Anemia was found in $47 \%$ of children and the mean of hemoglobin was 11.4 \pm 1.6 . The correlation between anemia in children and disease was found to be statistically not significant when chi square test was done ( $\mathrm{p}$-value $=0.148)$.

Table 5: The correlation between age and diseases among children

\begin{tabular}{|l|l|l|l|l|}
\hline Children & oral cancer\% & cleft lip/palate\% & Trauma\% & Others\% \\
\hline$<5$ & 0 & 88.9 & 0 & 11.1 \\
\hline $5-11$ & 1.8 & 71.4 & 5.4 & 21.4 \\
\hline $12-14$ & 0 & 50 & 0 & 50 \\
\hline
\end{tabular}

Pearson chi2(6) $=33.163 \mathrm{Pr}=0.000$ 


\section{Discussion}

Data on the epidemiology of anemia in hospitalized patients in Sudan is scarce and among maxillofacial patients is almost lacking, most of the studies were children and pregnancy related. This was the first study conducted at Khartoum Teaching Dental Hospital (KTDH) to assess the hemoglobin level among patients undergoing surgery under general anesthesia.

In the present study anemia was found in about half of the patients admitted at KTDH. Anemia was found in $48.2 \%$ of males, $45 \%$ of females and in $47 \%$ of children. In 2004, a systematic review done by Shander et al, revealed that the prevalence of preoperative anemia ranges from $5 \%$ to $75 \%$ depending on patient susceptibility and the proposed surgical procedure(11). Another meta analysis by Gaskell et al, showed that the overall prevalence of anemia in the older population was $17 \%$ and as high as $40 \%$ in hospitalized patients(12). In USA ; Dunne et al, found that preoperative anemia is $33.9 \%(13)$, and Sahadevan et al, reported a prevalence of anaemia of more than $30 \%$ in a hospitalised population, with a large proportion being nutritional anemias (14), which is in agreement with what was found in the present study.

It's important to consider the prevalence of anemia in normal population in Sudan in order to justify the results of the present study. In 1995, nutritional anemia was among the ten major causes for hospital admission in Sudan (5). Munsoor et al, found that the mean hemoglobin level in Port Sudan city (2012-2014) is $10.6 \mathrm{mg} / \mathrm{dl}$ and $15.3 \%$ of the population was anemic. In kassala; eastern Sudan a study was done in 2011 by Abdalla et al, revealed high prevelance of anaemia among adults (36.2\%) regardless of their age, sex and educational level(6). Therefore it is not infrequent to find a patient with anemia among the hospitalized patients. Hemoglobin level was found to be different between the different age groups in the same category of patients. Our results were compatible with the findings in the literature regarding the relation between age and anemia; which is in agreement with the findings of Balducci et al, who reported increase in incidence and prevalence of anemia with increasing age (15).

What is in common among oral and maxillofacial patients are the eating and sometimes swallowing difficulties they face because of their oral diseases.There was significant association between age and disease. Oral cancer patients were among the old groups, trauma was in teenagers and young adults and cleft lip and palate were in younger age groups. In the present study one way analysis showed a significant difference in hemoglobin level between the four categories of patients.

\section{Conclusion}

Prevalence of anemia in patients admitted at KTDH was $48.2 \%$ for males, $45 \%$ for females and $47 \%$ for children. The results of the present study indicated the role of the underlying disease (oral cancer, cleft lip and palate, trauma and benign lesions) as a causative factor for anemia. Age was strongly associated with anemia. Anemia was found in approximately half of the patients admitted to KTDH and most of the time it was discovered during the preoperative investigations for surgery preparation.

\section{References}

[1]. nissenson ar, goodnough 1t, rw. d.anemia: not just an innocent bystander? .arch intern med 2003;163(12):1400-4.

[2]. clevenger b, t. r. pre-operative anaemia. anaesthesia.2015;70 suppl 1:20-8, e6-8.

[3]. auerbach m. intravenous iron in the perioperative setting. am j hematol. 2014;89(9):933.

[4]. dharmarajan ts, avula s, jayakrishnan 1, jospeh p, talamati j, ep. kmn. mild anemia increases the risk of falls in hospitalized older adults. . j am geriatr soc. 2005;53(s94).

[5]. munsoor mohammed munsoor, mohammed omer gibreel, mubarak el saeed el karsani. nutritional anemia among patients referred to hematology laboratories in port sudan city. clinical medicine journal, . 2015;1(2):38-42.

[6]. tajeldin $m$ abdallah ia, mutaz a abdelhadi, mohammed $f$ siddig and abdelaziem a ali. anaemia among adults in kassala, eastern sudan. bmc research notes. 2012;5:202.

[7]. sarar mohamed, mohamed diab hussein. prevalence of thinness, stunting and anemia among rural school-aged sudanese children: a cross-sectional study. j trop pediatr,. 2015.

[8]. donat r. spahn. anemia and patient blood management in hip and knee surgery. a systematic review of the literature. anesthesiology 2010;113:482-95.

[9]. vasudevaru, narayanan, bhattathiri. relation of erythrocyte and iron indices to oral cancer growth. radiotherapy and oncology. 2001 may;59(2):221-6.

[10]. izaks gj, westendorp, r.g.j. \& knooks, d.1. (1999) the definition of anaemia in older persons. journal of the amer can med cal assoc at on. $281,1714-1717$.

[11]. shander a, knight $\mathrm{k}$, thurer $\mathrm{r}$, adamson $\mathrm{j}$, r. s. prevalence and outcomes of anemia in surgery: a systematic re view of the literature. am j med 2004;116:58s-69.

[12]. gaskell h, derry s, andrew moore r, . mh. prevalence of anaemia in older persons: systematic review. bmc geriatr 2008; 8: 1 .

[13]. dunne j, et al. j surg res. 2002;102:237-44.

[14]. sahadevan s, choo pw, fj. j. anaemia in the hospitalised elderly. singapore med j 1995;36(4):375-8.

[15]. balducci 1. epidemiology of anemia in the elderly: information on diagnostic evaluation. . $j$ am ger soc. 2003;51(3):s2-9. 